\title{
Expansion of CCR5+ CD4+ T-lymphocytes in the course of active pulmonary tuberculosis
}

\author{
M.B. Santucci*, M. Bocchino", S.K. Garg*, A. Marruchella\#, V. Colizzi*, C. Saltini\#, M. Fraziano*
}

Expansion of CCR5+CD4+ T-lymphocytes in the course of active pulmonary tuberculosis. M.B. Santucci, M. Bocchino, S.K. Garg, A. Marruchella, V. Colizzi, C. Saltini, M. Fraziano. (C) ERS Journals Ltd 2004.

ABSTRACT: Nonsyncytium inducing, macrophage tropic HIV strains predominate in the course of active tuberculosis (TB). The present study assesses the expression of CCR5 in CD4+ T-lymphocytes from blood and bronchoalveolar lavage (BAL) of TB patients, non-TB lung disease controls and healthy controls.

Memory $($ CD45RO +$)$, recently activated $(\mathrm{CD69}+)$, proliferating $(\mathrm{Ki67}+) \mathrm{CCR5}+$ or CCR3 + CD4+ T-lymphocytes were determined by multiparametric flow cytometry analysis.

Results show that BAL CD4+ T-lymphocytes expressing CCR5 or CCR3 were significantly increased when compared to peripheral blood both in patients and in healthy controls. However, the data show that the proportions of peripheral blood CCR5+ CD4+ and CCR3+ CD4+ T-lymphocytes and BAL CCR5+ CD4+ Tlymphocytes, but not BAL CCR3 + CD4+ T-lymphocytes, were significantly increased in TB patients. Furthermore, the observation that BAL CCR5+ CD4+ T-lymphocytes from TB patients expressed early activation markers, were not proliferating and showed down-regulation of CCR5 expression suggests recruitment and trapping at the site of disease.

Altogether, these results suggest that the lower respiratory tract mucosa may provide cellular targets accessible for efficient transmission of macrophage tropic HIV-1 variants and that tuberculosis may enhance this phenomenon.

Eur Respir J 2004; 24: 638-643.
*Dept of Biology, University of Roma "Tor Vergata", and "Division of Respiratory Medicine, University of Roma "Tor Vergata" at the INMI L. Spallanzani-IRCCS, Roma, Italy.

Correspondence: M. Fraziano, Dept of Biology, University of Roma "Tor Vergata", Via della Ricerca Scientifica 00133, Roma, Italy. Fax: 39672594224

E-mail: fraziano@bio.uniroma2.it

Keywords: CCR3

CCR5

lymphocytes

Mycobacterium tuberculosis

tuberculosis

Received: September 162003

Accepted after revision: May 282004

The present study was supported in part by Italian Ministry of University (MIUR 60\%) and by the IV Research Program on AIDS (Grant N. 50D.05) of the Italian Institute of Health. S.K. Garg was supported by a UNESCO-ROSTE study fellowship.
Tuberculosis (TB), one of the most frequent AIDS-defining complications of HIV infection [1], represents one of the major risk factors for AIDS progression, as infection with Mycobacterium tuberculosis results in enhanced susceptibility of CD4+ cells to HIV infection, both in terms of viral entry and replication [2-3]. In vitro, co-infection of monocytederived macrophages with $M$. tuberculosis and HIV is known to increase both viral replication and HIV transmission to T-cells [4]. At the molecular level, it has been shown that $\mathrm{I} \kappa \mathrm{B}-\alpha$, the major cytoplasmic inhibitor of the nuclear factor$\mathrm{kB}$, is constitutively degraded in freshly isolated peripheral blood mononuclear cells (PBMC) and monocytes from patients with active TB [5]. In addition, the increased production of the pro-inflammatory cytokines tumour necrosis factor (TNF)- $\alpha$ and interleukin (IL)-6 during the course of TB may also play a pathogenic role by generating a microenvironment enhancing the productive infection of local lymphocytes [6].

The chemokine receptors CCR5 and CXCR4 have been shown to be the main co-receptors for macrophage-tropic (now identified as R5) and T-cell line-tropic (now identified as X4) HIV-1 isolates, respectively. Furthermore, other chemokine receptor molecules, such as CCR2b, CCR3, CCR8, CCR9, CX3CR1 (formerly designated CMKBRL1 or V28), the chemokine receptor-like orphan molecules STRL33/Bonzo, GPR15/BOB and Apj, may function as
HIV co-receptors [7]. M. tuberculosis infection increases cell expression of CCR5 both in vitro and in vivo, thereby enhancing susceptibility of cells of the monocyte/macrophage lineage to HIV infection [8]. An increase of both CCR5 and CXCR4 expression in peripheral CD4+ T-lymphocytes from active TB patients has been described [9], and CCR5 has also been identified as the main co-receptor for nonsyncytium inducing (NSI) HIV-1 subtype-C isolates from patients with active TB [10].

Previous studies have shown that the lung is a site of accumulation of activated memory cells expressing lateactivation cell surface markers [11], and that during the course of lung immune reactions the compartmentalised activation and proliferation of lung T-cells [12] leads to an exaggerated accumulation of memory T-cells [13], possibly through enhanced recruitment of activated memory T-cells from the blood [14]. Chemokine receptors are known to be differentially expressed on memory CD4+ T-cells depending on their polarisation [15]: T-helper (Th)1 lymphocytes express CCR 5 and CXCR3, whereas Th2 lymphocytes express CCR3, CCR 4 and CCR8. As Th1 lymphocytes expand in the lungs of patients with active tuberculosis [15], the present study was designed to analyse the expression of CCR 5 and CCR 3 on CD4+ T-cells derived from peripheral blood and from the lower respiratory tract of TB patients, non-TB lung disease controls and healthy controls. 


\section{Methods}

\section{Study subjects}

Ten subjects with pulmonary mycobacterial disease (nine with culture-confirmed pulmonary TB and one with cultureconfirmed $M$. avium lung disease) were enrolled in the study before the initiation of anti-mycobacterial treatment (age 36.6 \pm 15.2 yrs; three males; five Caucasians). Twelve subjects with non-TB lung diseases (three with interstitial lung disease, four with lung cancer, one with lung lymphoma, three with bacterial pneumonia, one with atypical pneumonia; age 66.1 \pm 8.9 yrs; nine males; 11 Caucasians) were enrolled as disease controls. Six subjects undergoing bronchoscopy and bronchoalveolar lavage (BAL) for the diagnosis of nodular lesions (age $57.6 \pm 12.8$ yrs; one male; six Caucasians) were recruited in the study as healthy controls, as their BAL showed no bacteriological or cytological abnormalities. Final diagnoses were fibrocalcific TB sequelae in three cases presenting with haemoptysis (two cases) and dry cough (one case), lung sequestration in one case, hamarto-chondroma in one case, and a false image on chest radiography in one case. Subjects were enrolled in the study after informed consent (INMI Lazzaro Spallanzani IRCCS Ethics Committee).

\section{Blood and lung cells}

Whole blood was collected by venipuncture and PBMC were separated by centrifugation on a Ficoll-Hypaque gradient as previously described [8]. BAL was carried out during diagnostic fibreoptic bronchoscopy (Olympus BF 30; Olympus, Lake Success, NY, USA), following local anaesthesia with $2 \%$ lidocaine by instillation of three $50-\mathrm{mL}$ aliquots of $0.9 \%$ saline in a subsegmental bronchus, followed by low-pressure aspiration. BAL fluids were filtered through a monolayer of gauze, cells were collected by centrifugation at $350 \times g$ for $10 \mathrm{~min}$ and suspended in PBS, $0.5 \% \mathrm{BSA}, 0.1 \%$ $\mathrm{NaN}_{3}$ solution for flow cytometric analysis.

\section{Flow cytometry}

BAL cells and PBMC were labelled with fluorochromeconjugated monoclonal antibodies directed against CD3 (FITC-conjugated, clone UCHT1, IgG1; Becton Dickinson, San Jose, CA, USA), CD4 (Cy-chrome-conjugated, clone RPA-T4, IgG1; Becton Dickinson), CD69 (FITC-conjugated, clone $\mathrm{CH} / 4$, IgG2a; Serotec, Cergy Saint-Christophe, France), CD45RO (FITC-conjugated, clone UCHL1, IgG2a; Serotec), CCR5 (PE-conjugated, clone 2D7, IgG2a; Becton Dickinson), CCR3 (PE-conjugated, clone 61828.111, IgG2a; R\&D Systems, Minneapolis, MN, USA). Premixed cocktails containing appropriate fluorochrome-conjugated monoclonal antibodies (Fluorescein, Phycoerythrin, Cy-chrome) were reacted with $50 \mu \mathrm{L}$ of either BAL or PBMC cell suspension on ice for $30 \mathrm{~min}$ in the dark. Cells were then washed twice with PBS, $0.5 \%$ BSA, $0.1 \% \mathrm{NaN}_{3}$ and fixed over-night at $4{ }^{\circ} \mathrm{C}$ with $1.5 \%$ paraformaldehyde in PBS, as previously reported [16]. To assess CD4+ T-lymphocyte in situ proliferation, cells were analysed for the expression of the nuclear antigen Ki67 using an anti-Ki67 antibody (FITC-conjugated, clone B56, IgG1; Becton Dickinson) or an irrelevant antibody (FITCconjugated, clone MOPC-21, IgG1; Becton Dickinson) as control, by intracytoplasmatic staining as previously described [17]. Briefly, after membrane staining, cells were fixed for 5 min with $4 \%$ paraformaldehyde in PBS, washed and incubated with anti-Ki67 monoclonal antibody for
45 min at room temperature in the dark. Cells then underwent two final washings before being resuspended in PBS and analysed using a FACSCalibur (BD Biosciences, San Jose, CA, USA) by CellQuest software (BD Biosciences).

\section{Statistical analysis}

Comparisons between groups were done using paired and unpaired t-tests as indicated.

\section{Results}

In order to see whether pulmonary mucosa represents a site for accumulation of CCR5+ CD4+ and of CCR3+ CD4+ T-lymphocytes, both T-cell subpopulations were studied in BAL and in peripheral blood from TB patients, non-TB lung disease controls and healthy controls. Results expressed in figure 1 show that the proportion of CCR5+ CD4+ and CCR3+ CD4+ T-cells was significantly increased in BAL samples in comparison with peripheral blood, both in TB patients, non-TB lung disease controls and healthy controls.

In agreement with previous reports on CCR 5 expression on blood T-cells in active TB [9], the proportion of peripheral blood CCR5+ CD4+ T-cells in TB patients was significantly higher when compared to both non-TB lung disease controls and healthy controls (fig. 2a). Similarly, the proportions of CCR3+ CD4+ T-cells in peripheral blood of the same patients were significantly higher (fig. 2b) than that detected in both non-TB lung disease patients and healthy controls. Interestingly, when comparison was carried out at pulmonary mucosal level, the CCR5-expressing CD4+ T-cell population was increased in the lower respiratory tract of TB patients in comparison to both non-TB lung disease controls and healthy controls (fig. 2c), whereas the proportion of the CCR3+ CD4+ T-cells was similar in the three study groups (fig. 2d). Quantitative analysis of fluorescence intensity showed that in TB patients CCR5 expression on alveolar, but not blood, CD4+ T-cells was significantly lower than that observed in healthy controls $(24.1 \pm 8.83$ versus $46.97 \pm 19.56, \mathrm{p}=0.006)$ but not than that observed in non-TB lung disease patients (24.1 \pm 8.83 versus $32.58 \pm 16.9)$.

With regard to chemokine receptor expression on cytotoxic T-cells, alveolar CD4-/CD3+ T (mostly CD8+)-cells from TB patients expressed CCR 5 at a higher percentage than controls (TB patients $65.87 \pm 25.31$, non-TB lung disease controls $7.46 \pm 4.34$, healthy controls 15.12 $\pm 10.96 ; \quad \mathrm{p}=0.005$ and $\mathrm{p}<0.001$ when compared with non-TB lung disease controls and healthy controls, respectively), but not CCR3 (TB patients 14.54 \pm 17.65 , non-TB lung disease controls $22.3 \pm 22.94$, healthy controls $20.97 \pm 12.51$ ). No differences were observed in blood CD4-/CD3+ T-cells from TB patients and controls in terms of the proportion of either CCR 5+ cells (TB patients $1.03 \pm 1.84$, non-TB lung disease controls $0.72 \pm 0.73$, healthy controls $1.25 \pm 1.33$ ) or CCR3+ cells (TB patients $1.02 \pm 0.41$, non-TB lung disease controls $1.32 \pm 0.64$, healthy controls $0.59 \pm 0.98$ ).

To characterise the activation phenotype of CCR5+ and CCR3+ CD4+ lung T-cells, the proportions of CD4+ CCR5+ and CCR3 + cells that expressed the memory T-cell marker CD45RO, the recent activation T-cell marker CD69 and the proliferation cell marker Ki67 were analysed. Results show that, in TB patients as well as in non-TB lung disease controls, lung CCR5+ CD4+ and CCR3+ CD4+ T-lymphocytes were largely comprised of late-activated, nonproliferating memory cells, as all of them, both CCR5+ CD4+ and CCR3+ CD4+, expressed the CD45RO-positive memory T-cell phenotype 

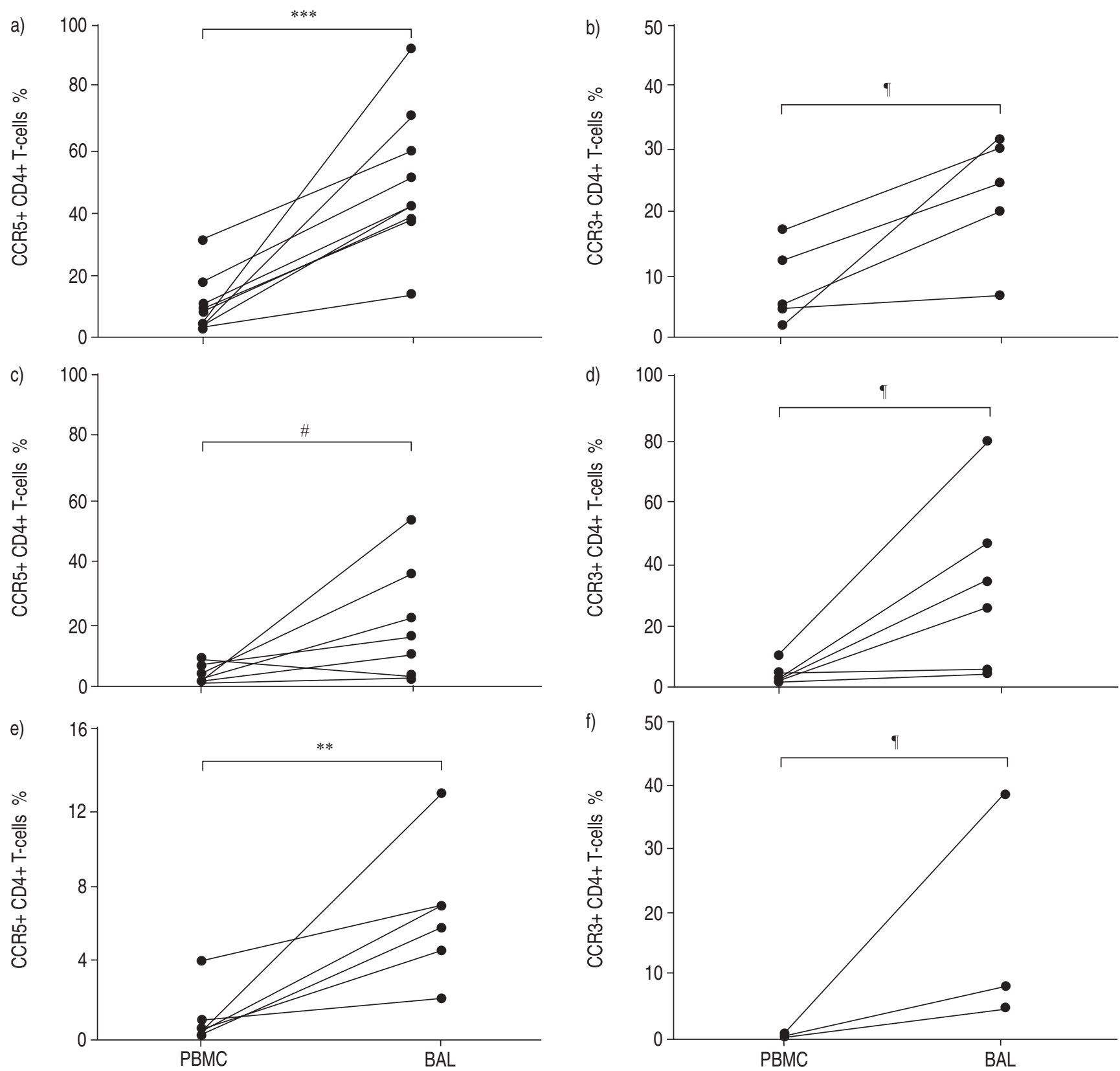

Fig. 1. - Proportion of bronchoalveolar lavage (BAL)- and peripheral blood mononuclear cell (PBMC)-derived CCR5+ CD4+ and CCR3+ CD4+ T-cells from tuberculosis (TB) patients ( $\mathrm{a}$ and $\mathrm{b}$, respectively), non-TB lung disease patients ( $\mathrm{c}$ and $\mathrm{d}$, respectively) and healthy controls ( and $\mathrm{f}$, respectively). ${ }^{* *}: \mathrm{p}=0.01 ;{ }^{* * *} \mathrm{p}=0.001{ }^{*}: \mathrm{p}=0.02 ;{ }^{\uparrow}: \mathrm{p}=0.03$, calculated by comparing differences between means by paired $\mathrm{t}$-test.

(table 1), while only $3.7 \pm 4.6$ of CCR5 and $12.85 \pm 12.98$ of CCR3 expressed the Ki67 proliferation marker $(\mathrm{p}<0.001$ and $\mathrm{p}<0.001$ as compared to CDR45RO+, respectively).

\section{Discussion}

Chemokine receptors play a key role in the process of HIV entry into target cells [6]. In this context, it has previously been shown that in vitro $M$. tuberculosis infection of monocyte-derived macrophages enhances the expression of the CCR5 chemokine receptor, similarly to what may be observed on ex vivo alveolar macrophages obtained from active TB patients [8]. In the present study, it has been shown that CCR5- and CCR3-expressing CD4+ T-lymphocytes compartmentalise to the lower respiratory tract and that, consistent with the observation that lung CD4 T-cells in active TB are almost entirely comprised of Th1 cells [18], CCR5-expressing CD4+ T-cells accumulate in the lung at higher rates in the course of active TB.

It has already been demonstrated that lung T-lymphocytes, both in normal individuals and in individuals with granulomatous diseases, are almost entirely comprised of $\mathrm{CD} 45 \mathrm{RO}+$ memory T-cells [13], and express both early- and lateactivation markers, such as CD69 and CD29 [19]. The data demonstrate that almost all of the CCR5+ and CCR3+ CD4+ T-cells compartmentalised to the lungs of patients with active TB express the memory T-cell phenotype, and that the majority may have been recently activated (as indicated by the expression of the CD69 marker), but are not proliferating in the lower respiratory tract milieu (as the expression of the Ki67 marker, a nuclear antigen expressed in all phases of the 

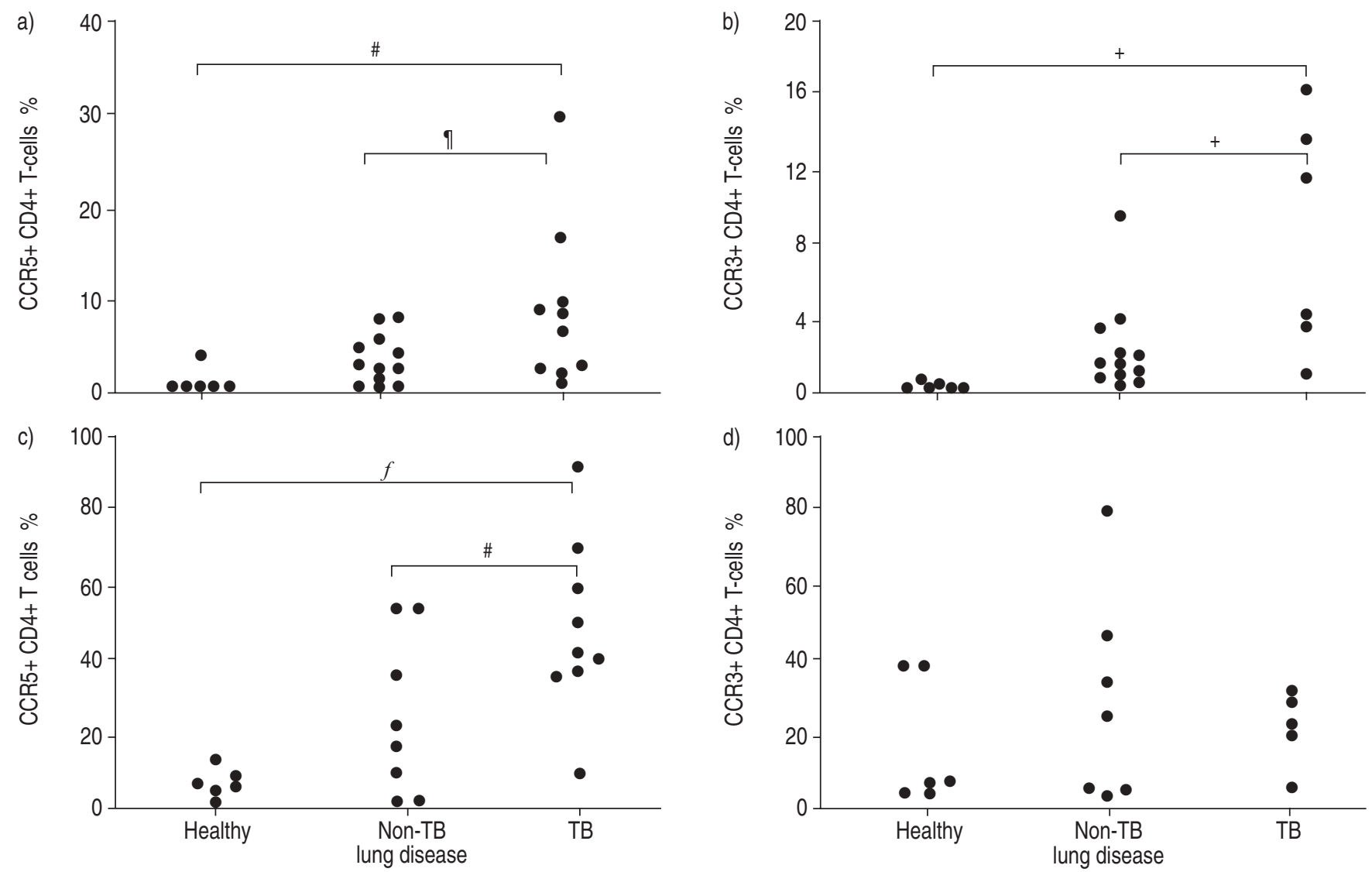

Fig. 2. - Proportion of CCR5+ CD4+ T-cells and CCR3+ CD4+ T-cells derived from peripheral blood mononuclear cells (a and b, respectively) and from bronchoalveolar lavage (c and d, respectively) from tuberculosis (TB) patients was compared with that observed in non-TB lung disease controls and healthy controls. ${ }^{\#}: \mathrm{p}=0.03$; ${ }^{\uparrow}: \mathrm{p}=0.04{ }^{+}: \mathrm{p}=0.008 ;{ }^{f}: \mathrm{p}=0.0006$, calculated by comparing differences between means by unpaired t-test.

Table 1.-Analysis of alveolar CD4+ T-cell subpopulation in tuberculosis (TB) patients and in non-TB lung disease controls

\begin{tabular}{lcclcc}
\hline & \multicolumn{2}{c}{ CCR5+ CD4+ T-cells } & & \multicolumn{2}{c}{ CCR3+ CD4+ T-cells } \\
\cline { 2 - 3 } \cline { 5 - 6 } & TB & non-TB & & TB & non-TB \\
\hline CD45RO+ & $95.0 \pm 3.8$ & $83.1 \pm 30.7$ & & $97.62 \pm 2.0$ & $80.7 \pm 33.1$ \\
CD69+ & $59.4 \pm 20.2$ & $81.6 \pm 23.5$ & & $65.06 \pm 26.0$ & $68.4 \pm 28.3$ \\
Ki67+ & $3.7 \pm 4.6$ & $18.6 \pm 19.0$ & & $12.85 \pm 13.0$ & $14.7 \pm 10.6$
\end{tabular}

Data are presented as mean $\pm \mathrm{SD}$ of the percentage of positive cells; due to the smaller number of cells recovered by bronchoalveolar lavage from healthy controls, this analysis was not carried out in these subjects.

cell cycle except G0 [20], was disproportionately lower than that of the CD69 and the CD45RO markers). Although it is not clear whether in situ activation occurs in the alveolar milieu of patients with TB [19], lung T-cells may be activated outside the lung in sarcoidosis, a granulomatous disorder involving primarily the lung, as CD45RA bright T-cells are rare [21] while IL-2 receptor-expressing CD4 T-cells are found in disproportionately high proportions in the bloodstream of patients with active disease [14]. In this context, the increased proportion of memory T-cells expressing CCR5 and CCR3, most notably of CCR5+ CD4+ T-cells, in the lungs of patients with active TB may be due to preferential recirculation and recruitment of recently activated memory cells to the mucosal compartment rather than to in situ proliferation and, possibly, to further activation in the lung. In this regard, this study shows that although the proportion of CD4+ CCR5+ T-cells was higher in the lungs of TB patients than controls, their CCR 5 expression was down-regulated in comparison to that of healthy controls, as indicated by mean fluorescence intensity analysis. This phenomenon may be explained by receptor internalisation after binding with chemokines present in the alveolar microenvironment in the course of TB. In this respect, macrophage inhibitory protein- $1 \alpha$ binding to CCR5 induces receptor internalisation and recycling, which are both regulated by actin polymerisation and activation of small G-proteins in a Rho-dependent manner [22]. However, the possibility of chemokine receptor shedding, as a further mechanism to explain the reduced membrane expression, cannot be excluded. In fact, evidence has shown that lipopolysaccharide- or TNF- $\alpha$-treated neutrophils downregulate CXCR1 and CXCR2 receptors through metalloproteinase (MMP) activation [23]. In this context, MMP are released from $M$. tuberculosis-infected macrophages both in vitro and in vivo, and they may contribute to cavity formation by their ability to digest collagens I-IV [24]. Although no evidence is actually available about a role of MMP in CCR5 downregulation, it is possible to hypothesise an MMP-mediated mechanism of CCR5 shedding in the course of $\mathrm{TB}$, which may contribute to the maintenance of lung inflammation. In fact, the reduced expression of certain inflammatory chemokine receptors (i.e. CCR1, CCR2 and 
CCR5) [25], possibly associated with receptor shedding, may deliver a stop signal to activated CCR5+ Th1 cells, which can then focus their action at sites of infection.

With the background that chemokine receptors play a major role in HIV infection, the very fact that the lung lymphocyte population is normally comprised of memory T-cells expressing the chemokine receptors CCR 5 and CCR3, and that in pulmonary TB the population of CCR5+ CD4+ activated T-cells expands in the lower respiratory tract, may render the lung a preferential target for the spread of the NSI macrophage-tropic viral variants, as alveolar and tissue lung macrophages may continuously transmit virus to susceptible antigen-specific CD4+ T-lymphocytes [3-4]. Consistently with this notion, NSI macrophage-tropic viral variants have been found in TB patients [10], where the infection of CCR5+ CD4+ T-cells is likely to lead to the deletion of $M$. tuberculosis-specific Th1 cells crucial for the anti-TB immune response [26], as vaginal CD4+ T-lymphocytes expressing high levels of CCR 5 are rapidly eliminated in the course of simian immunodeficiency infection [27]. The recent observation that the immunosuppressive drug rapamycin can downregulate CCR5 expression and inhibit R5 HIV entry into CD4 T-cells and macrophages [28] may open the way to new strategies to prevent the deletion of $M$. tuberculosisspecific lung memory T-cells.

Altogether, these results show that active tuberculosis, by driving the accumulation of CCR5+ CD4+ T-cells in the lower respiratory tract, may lead to an exaggerated expansion of a cell population highly susceptible to the transmission of nonsyncytium-inducing HIV-1 variants by alveolar macrophages and blood-derived monocytes. As the lung represents one of the preferential sites for HIV replication in the course of pulmonary tuberculosis in HIV-infected patients [29], the accumulation of CCR5+ CD4+ T-cell population may be responsible for the increase in HIV viraemia occurring in association with tuberculosis and favour the deletion of Mycobacterium tuberculosis -specific T-helper 1 cells.

\section{References}

1. Selwyn PA, Hartel D, Lewis VA, et al. A prospective study of the risk of tuberculosis among intravenous drug users with human immunodeficiency virus infection. $N$ Engl $\mathrm{J} \mathrm{Med}$ 1989; 320: 545-550.

2. Toosi Z, Sierra-Madero JG, Blinkhorn RA, Mettler MA, Rich EA. Enhanced susceptibility of blood monocytes from patients with pulmonary tuberculosis to productive infection with human immunodeficiency virus type 1. J Exp Med 1993; 177: 1511-1516.

3. Goletti D, Weissman D, Jackson RW, et al. Effect of Mycobacterium tuberculosis on HIV replication: role of immune activation. J Immunol 1996; 157: 1271-1278.

4. Mancino G, Placido R, Bach S, et al. Infection of human monocytes with Mycobacterium tuberculosis enhances human immunodeficiency virus type 1 replication and virus transmission to T cells. J Infect Dis 1997; 175: 1531-1535.

5. Lewin SR, Lambert P, Deacon NJ, Mills J, Crowe SM. Constitutive expression of $\mathrm{p} 50$ homodimer in freshly isolated human monocytes decreases with in vitro and in vivo differentiation: a possible mechanism influencing human immunodeficiency virus replication and mature macrophages. J Virol 1997; 71: 2114-2119.

6. Garrait V, Cadranel J, Esvant $\mathrm{H}$, et al. Tuberculosis generates a microenvironment enhancing the productive infection of local lymphocytes by HIV. J Immunol 1997; 159: 2824-2830.

7. Berger EA, Murphy PM, Farber JM. Chemokine receptors as HIV-1 coreceptors: roles in viral entry, tropism, and disease. Annu Rev Immunol 1999; 17: 657-700.
8. Fraziano M, Cappelli G, Santucci M, et al. Expression of CCR5 is Increased in human monocyte derived- and in alveolar- macrophages in the course of in vivo and in vitro Mycobacterium tuberculosis infection. AIDS Res Human Retroviruses 1999; 15: 869-874.

9. Jufferman NP, Speelman P, Verbon A, et al. Patients with active tuberculosis have increased expression of HIV coreceptors CXCR4 and CCR5 on CD+ T cells. Clin Infect Dis 2001; 32: 650-652.

10. Morrys L, Cilliers T, Bredell H, Phoswa M, Martin DJ. CCR5 is the major coreceptor used by HIV-1 subtype C isolates form patients with active tuberculosis. AIDS Res Human Retroviruses 2001; 17: 697-701.

11. Saltini C, Hemler ME, Crystal RG. T-lymphocytes compartmentalized on the epithelial surface of the lower respiratory tract express the very late activation antigen complex VLA-1. Clin Immunol Immunopath 1988; 46: 221-233.

12. Saltini C, Winestock K, Kirby M, Pinkston P, Crystal RG. Maintenance of alveolitis in patients with chronic beryllium disease by beryllium-specific helper T-cells. $N$ Engl J Med 1989; 320: 1103-1109.

13. Saltini C, Kirby M, Trapnell B, Tamura N, Crystal RG. Biased accumulation of T-lymphocytes with "memory"-type CD45 leukocyte common antigen gene expression on the epithelial surface of the human lung. J Exp Med 1990; 171: $1123-1140$.

14. Konishi K, Moller DR, Saltini C, Kirby M, Crystal RG. Spontaneus expression of the interleukin-2 receptor gene and presence of functional interleukin-2 receptors on $\mathrm{T}$ lymphocytes in the blood of individuals with active pulmonary sarcoidosis. J Clin Invest 1988; 82: 775-781.

15. von Andrian UH, Mackay CR. T-cell function and migration. Two sides of the same coin. N Engl J Med 2000; 343: $1020-1034$.

16. Santucci MB, Amicosante M, Cicconi R, et al. Mycobacterium tuberculosis-induced apoptosis in monocytes/macrophages: early membrane modifications and intracellular mycobacterial viability. J Infect Dis 2000; 181: 1506-1509.

17. Ciaramella A, Cavone A, Santucci MB, et al. Proinflammatory cytokines in the course of Mycobacterium tuberculosisinduced apoptosis in monocytes/macrophages. $J$ Infect Dis 2002; 186: 1277-1282.

18. Barry SM, Lipman MC, Bannister B, Johnson MA, Janossy G. Purified protein derivative-activated type 1 cytokineproducing CD4+ T lymphocytes in the lung: a characteristic feature of active pulmonary and nonpulmonary tuberculosis. J Infect Dis 2003; 187: 243-250.

19. Raju B, Tung CF, Cheng D, et al. In situ activation of helper $\mathrm{T}$ cells in the lung. Infect Immun 2001; 69: 4790-4798.

20. Gerdes J, Lemke H, Baisch H, Wacker HH, Schwab U, Stein $\mathrm{H}$. Cell cycle analysis of a cell proliferation associated human nuclear antigen defined by the monoclonal antibody Ki-67. J Immunol 1984; 133: 1710-1715.

21. Dominique S, Bouchonnet F, Smiejan JM, Hance AJ. Expression of surface antigens distinguishing "naïve" and previously activated lymphocytes in bronchoalveolar lavage fluid. Thorax 1990; 45: 391-396.

22. Mueller A, Strange PG. Mechanisms of internalization and recycling of the chemokine receptor, CCR5. Eur J Biochem 2003; 271: 243-252.

23. Khandaker MH, Mitchell G, Xu L, et al. Metalloproteinases are involved in lipopolysaccharide- and tumour necrosis factor- $\alpha$-mediated regulation of CXCR1 and CXCR2 chemokine receptor expression. Blood 1999; 93: 2173-2185.

24. Chang LC, Wysocki A, Tchou-Wong KM, Moskowitz N, Zhang Y, Rom WN. Effect of Mycobacterium tuberculosis and its components on macrophages and the release of matrix metallioproteinases. Thorax 1996; 51: 306-311.

25. Locati M, Otero K, Schioppa T, et al. The chemokine system: tuning and shaping by regulation of receptor expression and coupling in polarized responses. Allergy 2002; 57: 972-982. 
26. Rook GA, Hernandez-Pando R. The pathogenesis of tuberculosis. Annu Rev Microbiol 1996; 50: 259-284.

27. Veazey RS, Marx PA, Lackner AA. Vaginal CD4+ T cells express high levels of CCR5 and are rapidly depleted in simian immunodeficiency virus infection. J Infect Dis 2003; 187: 769-776.

28. Heredia A, Amoroso A, Davis C, et al. Rapamycin causes down-regulation of CCR5 and accumulation of anti-HIV $\beta$-chemokines: an approach to suppress R5 strains of HIV-1. Proc Natl Acad Sci USA 2003; 100: 10411-10416.

29. Nakata K, Rom WN, Honda Y, et al. Mycobacterium tuberculosis enhances human immunodeficiency virus-1 replication in the lung. Am J Respir Crit Care Med 1997; 155: 996-1003. 